\title{
FAILURE OF HYPOPHYSECTOMY TO AFFECT REGRESSION OF CYCLIC HAMSTER CORPUS LUTEUM
}

\author{
GILBERT S. GREENWALD \\ Departments of Obstetrics and Gynecology and Anatomy, \\ University of Kansas Medical Center, \\ Kansas City, Kansas, U.S.A.
}

(Received 5th February 1968)

The oestrous cycle of the hamster differs from the mouse and rat in two unique features: the extreme regularity of the 4-day cycle and the fact that the corpora lutea always begin to show signs of histological regression by Day 3 and have almost completely vanished by the next ovulation.

The rapidity of luteolysis raises the question whether luteal regression in the cyclic hamster is attributable to withdrawal of pituitary luteotrophic hormone(s) or to a surge in release of a hormone which directly destroys the corpus luteum. In the following experiment, these alternative possibilities were explored by hypophysectomizing hamsters on Day 1 of the cycle (metoestrus) and determining the subsequent fate of the corpora lutea in comparison with those in intact cyclic or pregnant animals. Serial sections of ovaries from three different hamsters were examined for each day; the ovaries were sectioned at $10 \mu$ and stained with haematoxylin and eosin. The diameter of four corpora lutea from each ovary was determined with an ocular micrometer by measuring and averaging two diameters at right angles to each other through the largest section of the corpus luteum (CL). The results are summarized in Table 1.

On Days 1 and 2, the cL of the cyclic and pregnant hamsters were the same size. Following hypophysectomy at Day 1 the GL on Day 2 were in the same size range as the other groups, but all antral follicles were atretic. On Day 3, the CL of the intact and hypophysectomized cyclic animals showed no further increase in diameter and incipient luteolysis was evident in the form of a leucocytic infiltration. In contrast, the CL of pregnancy on Day 3 were significantly larger than the CL of the oestrous cycle $(P<0.001)$ and were histologically normal. On Day 4, the CL of the intact and hypophysectomized hamsters had regressed similarly in size and this was reflected histologically by a similar degree of fatty degeneration. The CL of pregnancy continued their steady growth on Day 4.

The point of interest is that the corpora lutea regressed at the same time in cyclic hamsters regardless of whether the pituitary was present or not. The turning point in luteal development occurred at Day 3, when the GL of pregnancy continued to grow whereas the cL of the other groups commenced to involute. It thus appears likely for the hamster that the corpus luteum of the oestrous cycle is merely the histological consequence of ovulation and that it is a 


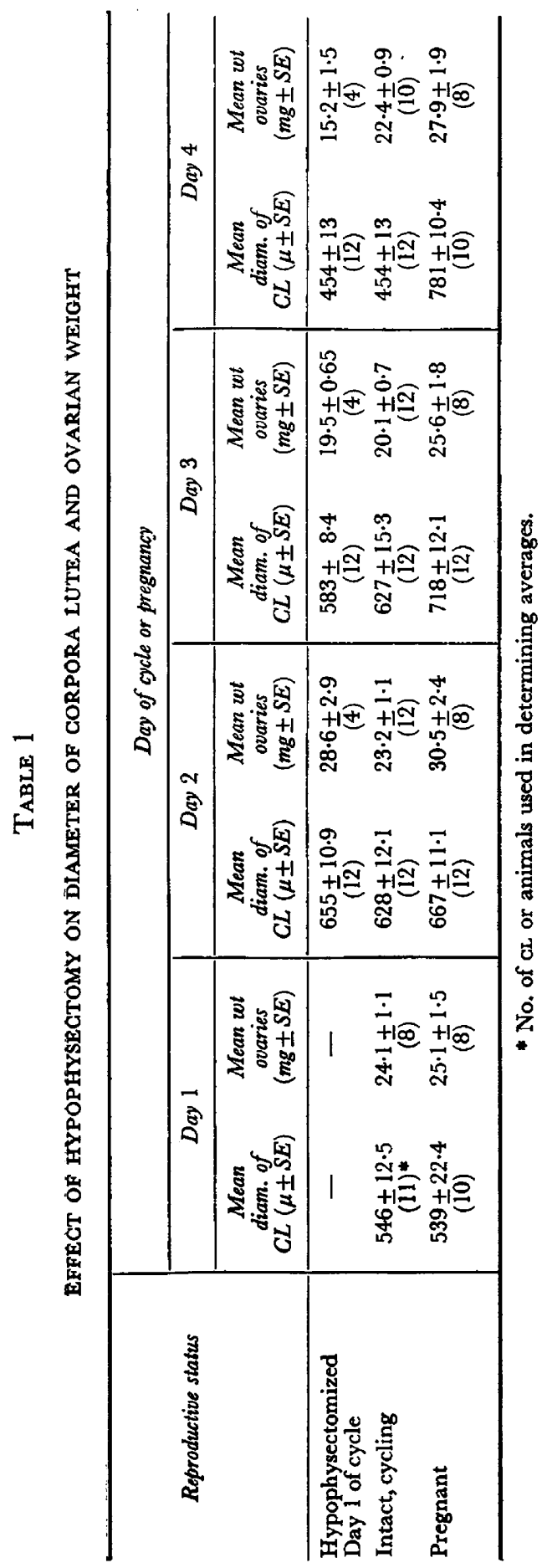


structure with limited-if any-ability to secrete progesterone. This could account for the extreme regularity of the oestrous cycle.

Recent work from this laboratory has shown that the maintenance of functional corpora lutea in the hamster depends on a minimal complement of prolactin and FSH (see Choudary \& Greenwald, 1968, for references). The fact that the CL regresses at the same time in both intact and hypophysectomized animals indicates that its involution is due to lack of luteotrophic stimulation rather than to the direct effect of a luteolytic hormone.

It is noteworthy that the regression of the cyclic cL on Day 3 is paralleled by a halving in the number of large follicles between Days 3 and 4 (Greenwald, 1961). At this stage of the cycle, deficiency in circulating levels of FSH seems to be the common denominator for both luteolysis and follicular atresia. It is therefore of interest that in mated hamsters there is no reduction in follicular numbers between Days 3 and 4 of pregnancy (Greenwald, 1964). This suggests that increasing circulating levels of $\mathrm{FSH}$, in addition to contributing to luteal maintenance, serve in an ancillary role to prevent follicular atresia.

This is a contribution from The Research Professorship in Human Reproduction. The work was supported by grants from the NIH, USPHS (HD 00 596) and the Ford Foundation.

\section{REFERENCES}

Ghoudary, J. B. R. \& Greenwald, G. S. (1968) Comparison of the luteolytic action of LH and estrogen in the hamster. Endocrinology (In press).

Greenwald, G. S. (1961) A quantitative study of follicular development in the ovary of the intact or unilaterally ovariectomized hamster. 7. Reprod. Fert. 2, 351.

Greenwald, G. S. (1964) Ovarian follicular development in the pregnant hamster. Anat. Rec. 148, 605. 